\title{
Consolidação de pastas cimentícias contendo policarboxilatos um estudo calorimétrico e reológico
}

\section{(Consolidation of policarboxilates containing cement pastes: a calorimetric and rheological study)}

\author{
J. S. Lyra', R. C. O. Romano ${ }^{2}$, R. G. Pileggi ${ }^{2}$, D. Gouvêa ${ }^{1}$ \\ ${ }^{1}$ Departamento de Engenharia de Materiais e Metalurgia, ${ }^{2}$ Departamento de Construção Civil, Escola \\ Politécnica, Universidade de S. Paulo, Av. Prof. Almeida Prado, Travessa 2, 83, S. Paulo, SP 05424-970 \\ dgouvea@usp.br
}

\begin{abstract}
Resumo
A utilização de policarboxilatos em concretos é pratica comum no setor de construção civil, visto que esses aditivos auxiliam na dispersão das partículas, possibilitam a redução da quantidade de água de amassamento, permitem melhorar as propriedades no estado fresco e endurecido. Entretanto, é sabido que apesar de possibilitar a produção de concretos de alto desempenho, a presença dos policarboxilatos pode afetar a cinética de reação do cimento e de aglomeração das partículas durante a consolidação. Ao mesmo tempo em que o fluxo de calor liberado durante a reação pode ser quantificado em calorímetros de condução isotérmica, a aglomeração pode também, ser acompanhada a partir de ensaios reológicos oscilatórios com controle de freqüência e deformação. Os resultados obtidos separadamente, quando avaliados conjuntamente podem melhor ilustrar o efeito da presença dos aditivos durante o endurecimento do cimento, abordagem que é apresentada como objetivo principal deste trabalho. Para tanto, pastas cimentícias formuladas com CPIIE-32 foram avaliadas em função da variação do teor de policarboxilato e os resultados mostraram que a taxa de consolidação (promovida pela ação conjunta da reação química e da aglomeração) apresenta relação direta com o teor do aditivo.
\end{abstract}

Palavras-chave: policarboxilato, pasta cimentícia, reação de hidratação, aglomeração, consolidação.

\begin{abstract}
Polycarboxylates are frequently used as additives to concrete production in the construction industry. The additive increases the dispersion of the particles, reduces the amount of water, and improves the mechanical properties of concrete in the fresh and settled state. However, although it is well known that polycarboxylate additives enable the production of high performance concretes, their presence may affect on both kinetics and thermodynamics of cement's particles during hydration reaction through consolidation. While the flow of heat released by the hydration reaction can be measured by isothermal calorimetry, concrete consolidation can be measured by oscillatory rheometry. The results obtained separately, when considered together could better illustrate the effect of the additives on the cement setting. This was the main subject in this work. Therefore, cement pastes containing commercial CPIIE-32 were evaluated by simultaneous heat of hydration and rheological measurements with different amounts of polycarboxylate. The results obtained separately, when considered together can better illustrate the effect of additive content during the hardening, and this approach is presented as a main objective of this work. For this, cement pastes formulated with CPII-32 were evaluated according to the variation of the content of polycarboxylate and the results showed that the rate of consolidation (promoted by the joint action of the chemical reaction and particle agglomeration) has direct relation with the level of the additive.
\end{abstract}

Keywords: polycarboxylate, cement paste, hydration reaction, agglomeration, consolidation.

\section{INTRODUÇÃO}

Os aditivos a base de policarboxilato são amplamente utilizados no setor construtivo com o objetivo de melhorar as características dos materiais cimentícios tanto no estado fresco quanto endurecido [1-5].

Conhecidos também como dispersantes, superplastificantes ou, ainda, plastificantes de $3^{\text {a }}$ geração, os policarboxilatos são agentes ativos de superfície que atuam impedindo, ou pelo menos dificultando, a aproximação das partículas mais finas da composição dos concretos, de acordo com o mecanismo de estabilização eletroestérica $[6,7]$. Com isto, permitem reduzir a viscosidade das suspensões, possibilitam aumentar o teor de sólidos ou reduzir a quantidade de água de amassamento (podendo chegar em até $40 \%$ ), melhoram a trabalhabilidade e o desempenho dos materiais em uso $[1,2,5,8,9]$. Entretanto, a presença dos policarboxilatos pode alterar de forma indesejada a cinética de reação e a aglomeração das partículas de cimento durante a consolidação, caso, por exemplo, a dosagem seja realizada de forma excessiva [1, 10]. Além disto, como ainda não há um consenso geral 


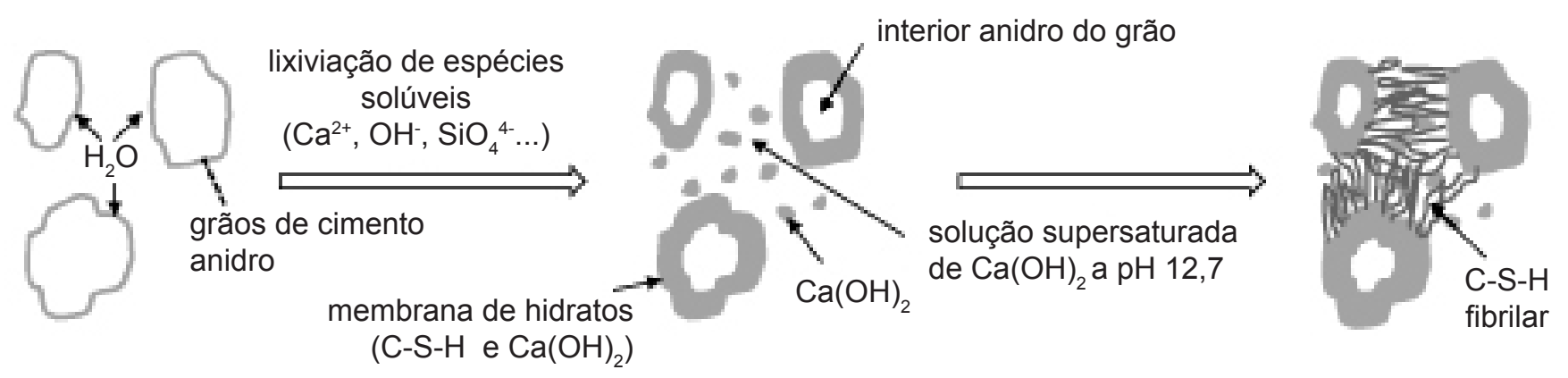

Figura 1: Ilustração das partículas de cimento em diferentes tempos de reação de hidratação [11].

[Figure 1: Illustration of the cement particles at different times of hydration reaction [11].]

que possibilite a correta e confiável otimização desses insumos em obra, muitas vezes os aditivos são dosados de forma empírica, com base na experiência do profissional. Quando o cimento é misturado com água uma série de reações simultâneas e consecutivas é iniciada, as quais são responsáveis pelo enrijecimento do material em função do tempo. Essas reações são conhecidas como hidratação e ocorrem basicamente segundo a ilustração esquemática da Fig. 1, adaptada [11].

Entretanto, diferentes mecanismos são propostos para descrever as reações de hidratação do cimento, mas é senso comum que as reações são continuas e exotérmicas e, podem ser divididas em alguns estágios de acordo com o fluxo de calor, conforme ilustrado na Fig. 2a [1, 12]. Na

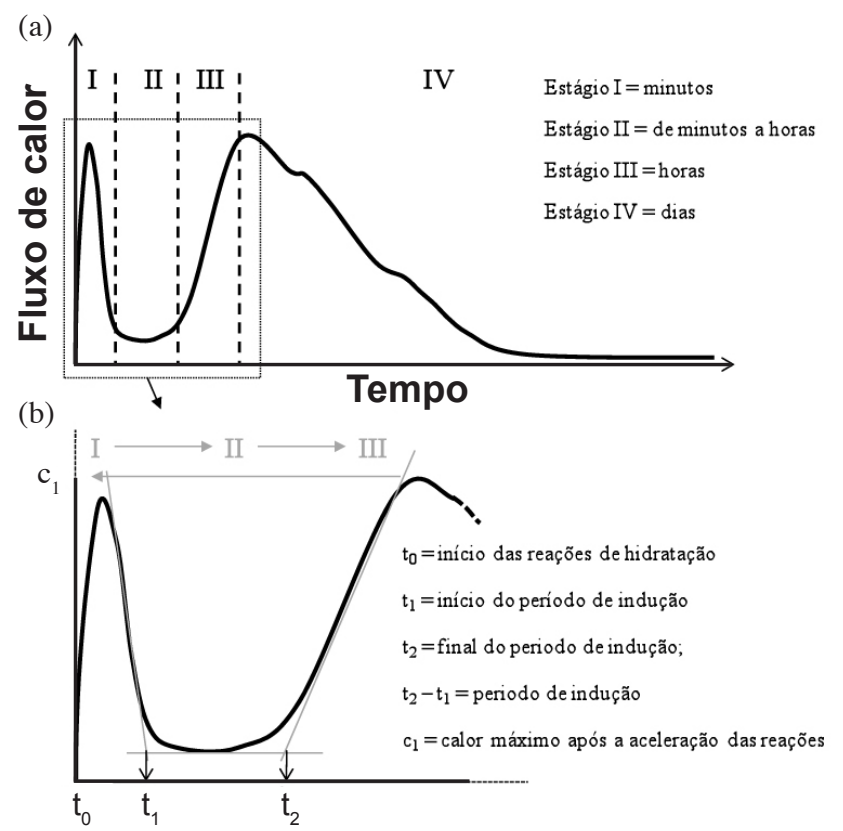

Figura 2: Perfil típico de fluxo de calor durante as reações de hidratação de um cimento Portland comum. Em (a) é ilustrada uma curva completa, com a reação acompanhada por dias e em (b) um zoom na região até o período de desaceleração das reações químicas [1].

[Figure 2: Flow pattern during the cement hydration reaction. In (a) is shown the flow heat followed by days and in (b) followed until the de-acceleration period [1].]
Fig. $2 b$ é mostrada uma curva representativa do fluxo de calor até o período de desaceleração das reações, ilustrando como os resultados serão tratados neste trabalho: $\mathrm{t}_{0}$ é o tempo de início da reação de hidratação, $\mathrm{t}_{1}$ é o início do período de indução, $\mathrm{t}_{2}$ é o final do período de indução, $\mathrm{t}_{2}-$ $\mathrm{t}_{1}$ é o tempo no período de indução e $\mathrm{c}_{1}$ equivale ao calor máximo após o período de aceleração das reações.

No estágio I, que ocorre em minutos, a rápida liberação de calor após o primeiro contato da água com o cimento, ocorre devido à dissolução dos álcalis ou sulfato de cálcio e íons como K+, $\mathrm{Na}^{+}, \mathrm{SO}_{4}{ }^{2-}, \mathrm{Ca}^{2+}$ [13-15]. No segundo estágio, conhecido como período de indução, as reações de hidratação ocorrem muito vagarosamente, devido à formação de uma camada de gel de C-S-H (silicato de cálcio hidratado) ao redor das partículas de cimento. De acordo com Thomas et at [16], a camada de gel de C-S-H formada na pasta consiste na agregação de partículas coloidais não estruturadas na superfície do cimento, formando uma camada de somente alguns nanômetros. Este estágio ocorre em um período que vai de minutos até horas, dependendo das características do cimento, adições ou aditivos. Quando a camada de gel é rompida, a difusão iônica é facilitada e a velocidade de reação aumenta. No estágio III, devido à saturação iônica da fase aquosa, ocorre a rápida formação do C-S-H e C-H (hidróxido de cálcio), resultando em redução gradual da concentração de íons $\mathrm{Ca}^{2+}$. Finalmente, no estagio IV a formação de C-S-H e C-H continua, mas de forma mais lenta, reduzindo a velocidade da reação global $[1,13,14]$.

É citado que o desenvolvimento das reações de hidratação estão diretamente relacionadas com a liberação dos íons $\mathrm{Ca}^{2+}$ na suspensão [17]. Entretanto, com a introdução do dispersante na formulação das suspensões, os íons $\mathrm{Ca}^{2+}$, liberados durante a reação de hidratação, são consumidos até que o aditivo perca sua eficiência. Com isso a hidratação é retardada, fato que pode afetar as características dos materiais nos estados fresco e endurecido e a produtividade em obra. Assim, este trabalho foi realizado com o objetivo de avaliar o efeito da variação do teor de policarboxilato no processo de consolidação do material cimentício, a partir de estudos da cinética de reação (componente química do processo) e da cinética de aglomeração (componente física). 


\section{PROCEDIMENTO EXPERIMENTAL}

\section{Materiais}

Foi utilizado um cimento à base de escória de alto-forno (CPIIE-32), fornecido por uma concreteira de S. Paulo, $\mathrm{SP}$, sendo, portanto, comumente empregado na formulação de concretos comerciais, e um aditivo comercial à base de policarboxilato de sódio (Glenium 51, Basf).

\section{Preparação das pastas}

As pastas cimentícias foram processadas com proporção entre água e cimento de 0,38 em um misturador de bancada com hélice naval, Labortechnik RW20-IKA. A água e o cimento foram adicionados em um béquer e a mistura foi realizada inicialmente sob rotação $300 \mathrm{rpm}$ por $2 \mathrm{~min}$. Em seguida a velocidade foi aumentada para $600 \mathrm{rpm}$, na qual foi mantida por mais $2 \mathrm{~min}$. Após a mistura, a pasta foi dispersa em um dispersor de alta energia de cisalhamento, in line mixer, IKA- Labortecknik T25 basic, por $5 \mathrm{~min}$, sob rotação de $9400 \mathrm{rpm}$. Para garantir que a mesma quantidade de água utilizada na pasta de referência fosse mantida nas pastas aditivadas, foi descontada a quantidade de líquido advinda do dispersante. Por isso, como todas as suspensões foram preparadas com $50 \mathrm{~g}$ de cimento, em todos os casos a quantidade de liquido foi de $19 \mathrm{~g}$.

\section{Ensaios}

Distribuição granulométrica a laser: a caracterização granulométrica do cimento foi realizada em um granulômetro a laser Malvern Mastersizer S long bed Ver 2.19, com faixa de 0,05 a $555 \mu \mathrm{m}$.

Difração de raios X: a composição mineralógica dos materiais foi determinada em um difratômetro de raios $\mathrm{X}$ Bruker AXS D8 Advance, com radiação k $\alpha$ do cobre $(\lambda=1,5404 \AA)$. Os dados foram obtidos na faixa de $2 \theta$ entre $3^{\circ}$ e $70^{\circ}$, com passo de $0,02^{\circ} / \mathrm{seg}$, com $30 \mathrm{rpm}$ de rotação da amostra.

Área de superfície específica: a medida, baseada na adsorção física e dessorção de gás na superfície da amostra sólida, foi realizada a partir do método de BET (Brunauer, Emmet e Teller), em um equipamento Gemini 2375, Micromeritics com pré-tratamento das amostras a $200{ }^{\circ} \mathrm{C}$ e $100 \mathrm{mmHg}$.

Picnometria de gás hélio: a densidade real dos pós foi determinada por picnometria de gás He em um equipamento Multipicnometer Quantachrome MVP 5DC.

Calorimetria de condução isotérmica: Foi utilizado um calorímetro isotérmico, TA Instr. TAM Air, com precisão de $\pm 20 \mu \mathrm{W}$, com 8 canais para realização de ensaios paralelamente. O fluxo de calor foi acompanhado por $48 \mathrm{~h}$.

Reometria oscilatória: os ensaios oscilatórios foram realizados em um aparato de geometria de placas paralelas em um reômetro AR550, TA Inst.. Aproximadamente 2 $\mathrm{mL}$ da pasta foi colocado no equipamento e a amostra foi submetida a um esmagamento entre as placas paralelas até atingir o espaçamento de $1 \mathrm{~mm}$ (gap). Os ensaios de varredura de tempo foram realizados com preciso controle da temperatura em $25^{\circ} \mathrm{C}$, deformação de $10^{-4}$ rad e freqüência de $1 \mathrm{~Hz}$. Todos os ensaios tiveram a cinética de aglomeração acompanhada por $2 \mathrm{~h}$.

\section{RESULTADOS E DISCUSSÃO}

\section{Caracterização do cimento}

O cimento CPIIE-32 utilizado no trabalho apresenta a

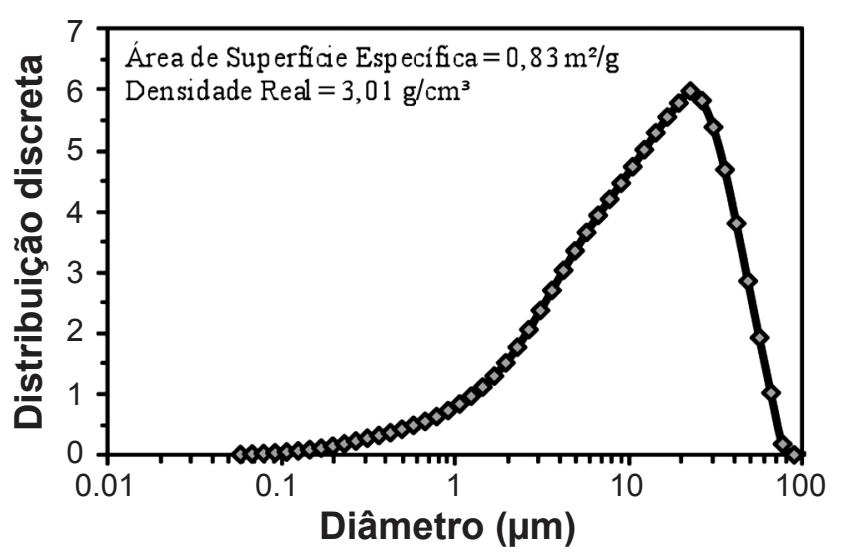

Figura 3: Distribuição granulométrica e características físicas das partículas do cimento.

[Figure 3: Granulometric distribution and physical properties of the cement.]

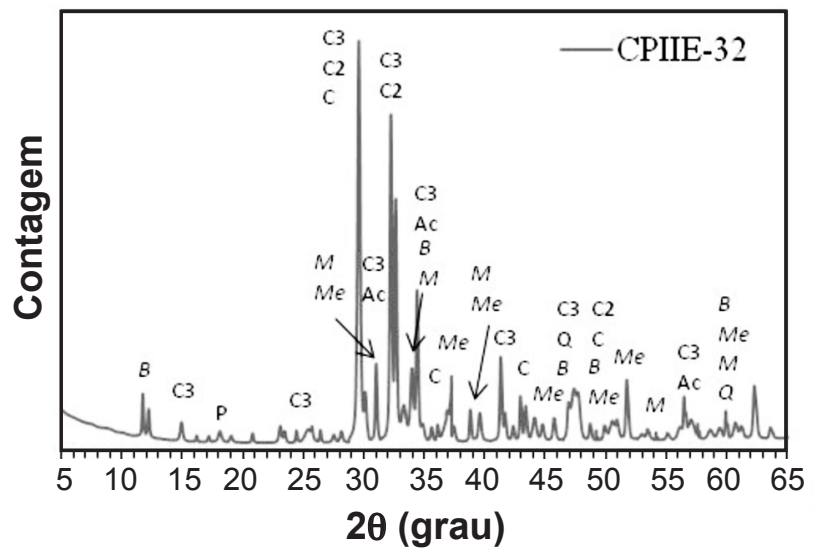

\begin{tabular}{|c|c|c|}
\hline Composto químico & Fórmula química & Mineral \\
\hline 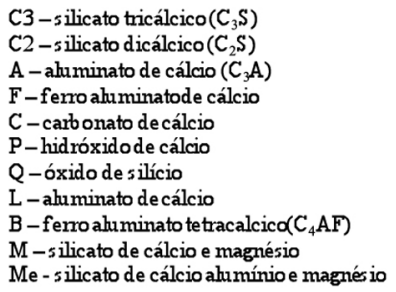 & $\begin{array}{c}\mathrm{Ca}_{3} \mathrm{SiO}_{5} \\
\mathrm{Ca}_{2} \mathrm{SiO}_{4} \\
\mathrm{Ca}_{3} \mathrm{Al}_{2} \mathrm{O}_{6} \\
\mathrm{Ca}_{12} \mathrm{Al}_{9} \mathrm{Fe}_{4} \mathrm{O}_{32} \\
\mathrm{CaCO}_{3} \\
\mathrm{Ca}(\mathrm{OH})_{2} \\
\mathrm{SiO}_{2} \\
\mathrm{Ca}_{3} \mathrm{Al}_{10} \mathrm{O}_{18} \\
4 \mathrm{CaO}_{2} \mathrm{Al}_{2} \mathrm{O}_{3} \mathrm{Fe}_{2} \mathrm{O}_{3} \\
\mathrm{Ca}_{3} \mathrm{MgO}_{8} \mathrm{Si}_{2} \\
\mathrm{Ca}_{8} \mathrm{~A}_{16} \mathrm{MgSi}_{5} \mathrm{O}_{28}\end{array}$ & $\begin{array}{c}\text { Alita } \\
\text { Belita } \\
- \\
- \\
\text { Calcita } \\
\text { Portlandita } \\
\text { Quatzo } \\
- \\
\text { Brownmilerita } \\
\text { Mervinita } \\
\text { Melilita }\end{array}$ \\
\hline
\end{tabular}

Figura 4: Composição mineralógica do cimento, obtida por difração de raios $\mathrm{X}$.

[Figure 4: Cement mineralogical composition, obtained by X-ray diffraction.] 
distribuição granulométrica e as propriedades físicas das partículas apresentadas na Fig. 3. Os valores apresentados são similares aos comumente encontrados em literatura, sendo que $90 \%$ das partículas do cimento utilizado apresentam diâmetros menores que $35 \mu \mathrm{m}$.

A área de superfície específica é uma propriedade que afeta diretamente o teor ótimo de aditivo, visto que para que ocorra de forma eficiente o mecanismo de estabilização da suspensão, o aditivo deve estar adsorvido por toda superfície do cimento.

A composição mineralógica é apresentada na Fig. 4. Como pode ser observado existe predominância das fases $\mathrm{C}_{3} \mathrm{~S}$ e $\mathrm{C}_{2} \mathrm{~S}$, mas há traços da fase $\mathrm{C}_{3} \mathrm{~A}$ e presença de brownmilerita, mervinita e melilita, características de cimento de escória.

\section{Cinética de reação - componente química da consolidação}

A hidratação do cimento é um processo complexo que normalmente resulta no endurecimento da suspensão: quando o cimento entra em contato com a água há formação

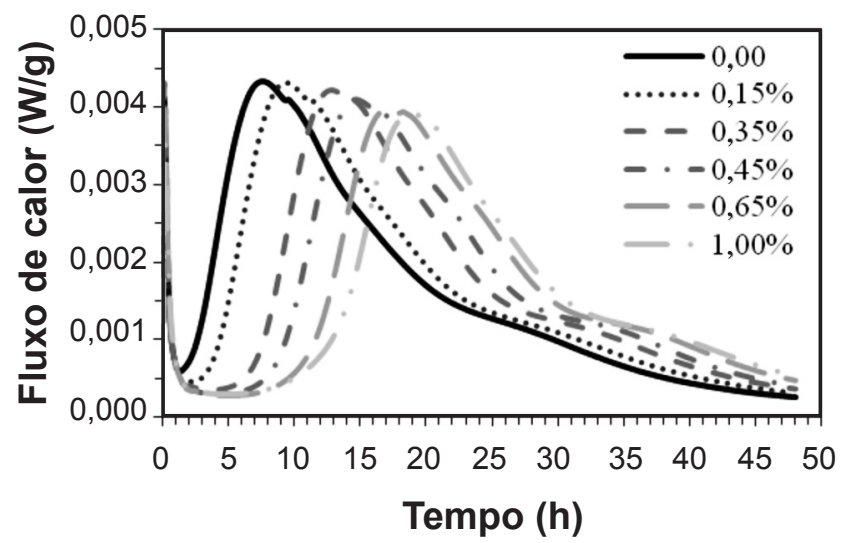

Figura 5: Fluxo de calor liberado durante a hidratação do cimento, nas pastas aditivadas com diferentes teores de policarboxilato.

[Figure 5: Flow heat during the cement hydration reaction as a function of the time for distinct polycarboxylate contents.] de diferentes compostos hidratados, dependentes da composição da matéria-prima e da presença de aditivos na formulação. No caso das pastas avaliadas, o aumento do teor de policarboxilato retardou a cinética de reação do cimento, apesar do início do período de indução ter sido independente do teor de aditivo e iniciar sempre em torno de $1 \mathrm{~h}$, o final foi distinto e dependente do teor (quanto maior o teor de superplastificante, maior o tempo de dormência - Fig. 5 e Tabela I).

Para ilustrar quantitativamente o efeito da variação do policarboxilato no tempo de indução, na liberação de calor e no calor total liberado após 48 h de reação, foi montada a Tabela I a partir da Fig. 5. Os dados foram tratados conforme apresentado anteriormente na Fig. 2.

O tempo no período de indução variou de $1: 10 \mathrm{~h}$ para a pasta isenta de aditivo até 11:30 h na pasta com o maior teor de policarboxilato utilizado no trabalho, fato que pode impactar diretamente na produtividade durante a execução da obra. Pode-se dizer que dois fenômenos são responsáveis por essa variação. No primeiro caso, a atuação dos grupos ionizáveis das moléculas poliméricas nas cargas superficiais das partículas diminui a concentração de $\mathrm{Ca}^{2+}$ em solução e torna a precipitação dos hidratos mais lenta. O segundo diz respeito à redução da taxa de dissolução do cimento anidro, por meio da formação de camadas adsorvidas na superfície das partículas de cimento, que dificultam a aproximação das moléculas de água [18]. É importante ressaltar que a presença de policarboxilato alterou somente a cinética de reação química do cimento, sendo que todas as reações que ocorrem na pasta pura acontecem nas pastas aditivadas em tempos maiores quanto maiores os teores de aditivos. As diferenças foram mais nítidas durante o período de indução e na etapa de aceleração das reações (no perfil de fluxo foram observadas distintas inclinações e picos com diferentes intensidades, em função do teor de dispersante - Fig. 5). Este fato pode ser enfatizado com a comparação do calor total acumulado após $48 \mathrm{~h}$ de reação, o qual foi reduzindo em função do aumento do teor do aditivo (Tabela I).

Os resultados apresentados até aqui ilustraram o impacto da variação do teor de policarboxilato na componente química da consolidação, variável que apesar de ser importante para

Tabela I - Resultados dos ensaios de calorimetria de condução isotérmica.

[Table I - Results of isothermal calorimetry test.]

\begin{tabular}{cccccc}
\hline $\begin{array}{c}\text { Teor de } \\
\text { policarboxilato } \\
(\%-p)\end{array}$ & $\begin{array}{c}\text { Tempo de } \\
\text { início da } \\
\text { indução }-\mathrm{t}_{1} \\
(\text { h:min })\end{array}$ & $\begin{array}{c}\text { Final do } \\
\text { período de } \\
\text { indução }-\mathrm{t}_{2} \\
(\text { h:min })\end{array}$ & $\begin{array}{c}\text { Tempo no } \\
\text { período de } \\
\text { indução }-\mathrm{t}_{2}-\mathrm{t}_{1} \\
(\text { h:min })\end{array}$ & $\begin{array}{c}\text { Pico de calor após } \\
\text { a aceleração das } \\
\text { reações } \\
\left(\times 10^{-3} \mathrm{~W} / \mathrm{g}\right)\end{array}$ & $\begin{array}{c}\text { Calor total } \\
\text { acumulado } \\
\text { após 48 } \mathrm{h} \\
(\mathrm{J} / \mathrm{g})\end{array}$ \\
\hline 0,00 & & $2: 10$ & $1: 10$ & 4,37 & 281 \\
0,15 & & $4: 00$ & $3: 00$ & 4,37 & 278 \\
0,35 & $1: 00$ & $7: 05$ & $6: 05$ & 4,20 & 272 \\
0,45 & & $8: 40$ & $7: 40$ & 4,10 & 268 \\
0,65 & $11: 00$ & $10: 00$ & 3,95 & 259 \\
1,00 & $12: 30$ & $11: 30$ & 3,90 & 246 \\
\hline
\end{tabular}


a compreensão do resultado global do enrijecimento do sistema cimentício, representa uma das possíveis causas do processo. Entretanto, o efeito gerado não pode ser discutido somente analisando-se esses resultados. Por outro lado, a cinética de aglomeração, componente física do processo de consolidação, pode ser avaliada a partir de ensaios quaseestáticos, como reometria oscilatória, e os resultados são apresentados a seguir.

\section{Cinética de aglomeração - componente física da consolidação}

O acompanhamento da componente física da consolidação é uma forma de quantificar o efeito da variação do teor de policarboxilato na aglomeração das partículas e os resultados são apresentados na Fig. 6 em função do módulo de armazenamento de energia elástica, G' . Nos baixos valores de G' a pasta apresenta comportamento de fluido viscoso e à medida que esse valor aumenta, amplia a tendência do comportamento do material como sólido elástico. Durante o ensaio foram impostas tensões de deformações dentro do limite de escoamento das pastas e, por isso, as estruturas geradas pela consolidação e hidratação não foram rompidas durante o ensaio. Assim, foi possível quantificar o real efeito do aditivo na aglomeração.

O cimento apresenta tendência natural de se aglomerar quando em contato com a água devido às interações de van der Waals e as forças eletrostáticas de atração, já que é composto por várias fases com cargas superficiais contrárias. As forças de aglomeração podem aumentar à medida que a hidratação do cimento prossegue devido ao aumento da força iônica e a formação dos produtos hidratados. Assim, qualquer fenômeno que afete a cinética de dissolução, a formação dos compostos hidratados, o movimento browniano das partículas em suspensão, a taxa de colisões entre elas e a velocidade da reação de hidratação do cimento, pode afetar a cinética de aglomeração. Por isto, na pasta isenta de aditivo a aglomeração ocorreu de

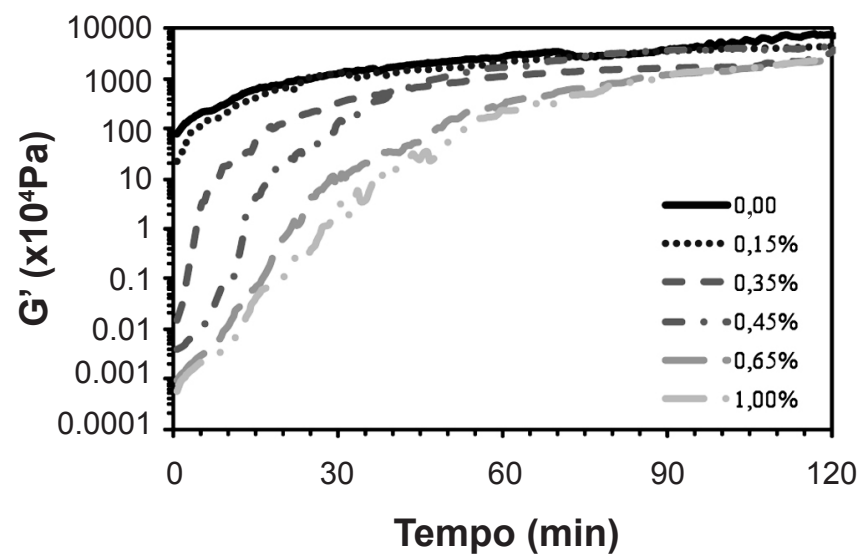

Figura 6: Cinética de aglomeração das pastas aditivadas com diferentes teores de policarboxilato.

[Figure 6: Kinetics of agglomeration as a function of the time for distinct polycarboxylate contents.] forma mais acentuada que nas pastas aditivadas, visto que não havia nenhum mecanismo de estabilização atuando no sistema, ou seja, um fenômeno que poderiam interferir na aglomeração não foi afetado e a atração entre as partículas foi favorecida. Já no caso das pastas aditivadas, mesmo a partir dos menores teores de aditivo, a cinética de aglomeração foi afetada, tornando-se mais lenta à medida que se aumentou o teor de policarboxilato. A presença do aditivo gerou o mecanismo de estabilização eletroestérico que dificultou a aproximação entre as partículas de cimento, provavelmente reduzindo a taxa de liberação de íons $\mathrm{Ca}^{2+}$ durante a hidratação e dificultando o aumento da força iônica do sistema. Também, com o aumento do teor de policarboxilato, maior quantidade do polímero permaneceu livre na suspensão e, por isso, maior tempo para o início da aglomeração foi observado, já que os íons cálcio gerados na dissolução poderiam ser instantaneamente consumidos pelo excesso de aditivo. De forma análoga ao que foi ilustrado para a componente química do processo de consolidação, o fenômeno de aglomeração permite quantificar somente a componente física do processo, evidenciando o efeito gerado pela presença e variação do teor de policarboxilato.

\section{Relação CAUSA - EFEITO durante a consolidação}

Avaliando-se conjuntamente os resultados obtidos nos diferentes ensaios, pode-se ter uma noção mais clara da relação causa-efeito no processo de consolidação das pastas cimentícias avaliadas em função do teor de dispersante. Assim, na Fig. 7 é apresentada à relação entre a componente química do processo, na forma de calor acumulado e a componente física, na forma de G'. A partir da análise conjunta dos resultados foi possível visualizar que a cinética de aglomeração e a cinética de reação química são fenômenos que ocorrem paralelamente e, que dependeram do teor de policarboxilato de sódio. Apesar de serem variáveis que

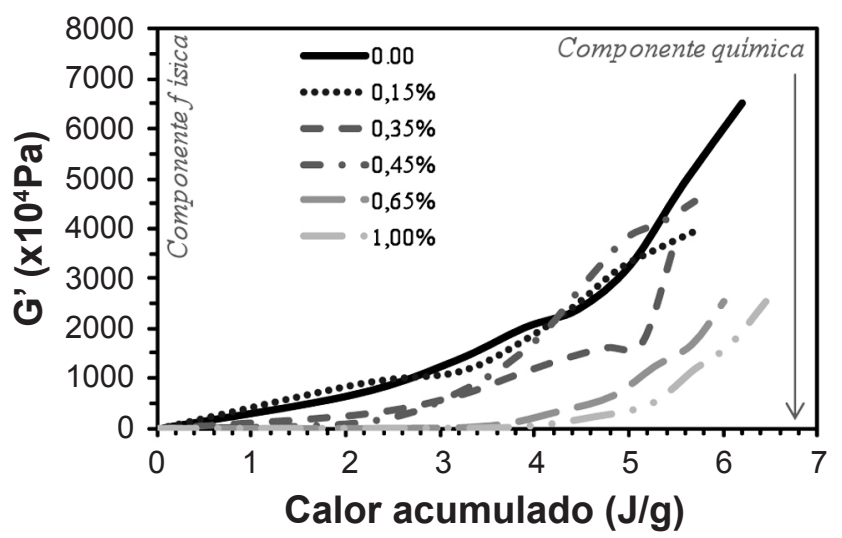

Figura 7: Relação causa-efeito no processo de consolidação, acompanhado a partir da cinética de reação química (calor acumulado) e cinética de aglomeração ( $\left.\mathrm{G}^{\prime}\right)$.

[Figure 7: Cause-effect relationship during the consolidation: cause - kinetics of chemical reaction (cumulative heat) and effect kinetics of agglomeration $\left(G^{\prime}\right)$.] 
atuam conjuntamente durante o processo de consolidação da suspensão cimentícia, a relação entre a parte física e a parte química do processo não foi linear. Com o aumento do teor de policarboxilato na composição foi preciso maior quantidade de calor (reagir mais) para se atingir a mesma aglomeração, medida na forma de G'. Assim, pode-se dizer que o impacto na cinética de hidratação foi maior que na cinética de aglomeração das partículas.

A presença do aditivo deve gerar um mecanismo de estabilização que dificulta a aproximação entre as partículas de cimento, mesmo quando a liberação de calor for elevada. Tal fato ilustra que o endurecimento do material cimentício não está relacionado somente com a reação química, mas também com as forças de superfícies incrementadas com a reação e com alterações no meio líquido. Pode-se dizer que a análise das alterações ocorridas durante a consolidação não é trivial, já que vários fenômenos físico-químicos estão ocorrendo ao mesmo tempo e a estrutura do cimento é alterada a todo instante. Isto significa que para estudos com outro tipo de cimento em associação com o mesmo aditivo ou, para o mesmo tipo de cimento em associação com outros tipos de aditivos, as tendências possivelmente não serão as mesmas que foram observadas neste trabalho. Por isso, a correta avaliação dos fenômenos que ocorrem durante o estado de transição entre um comportamento de fluido viscoso para um sólido elástico é de extrema importância para o desenvolvimento de materiais cimentícios com melhores propriedades e mais duráveis.

\section{CONCLUSÕES}

O aumento do teor de policarboxilato foi responsável pela alteração da cinética de reação do cimento. Apesar de não afetar o início do período de indução, o qual foi independente do teor e ocorreu em torno de $1 \mathrm{~h}$, aumentou o tempo no período de dormência, retardando o início da aceleração das reações. Enquanto na pasta não aditivada o tempo no período de dormência foi de 1:10 h, nas pastas aditivadas chegou a atingir até 11:30 h. Por consequiência, o calor total acumulado após $48 \mathrm{~h}$ de reação foi inversamente proporcional ao teor de policarboxilato. Da mesma forma que para a reação química, a componente física da consolidação foi afetada pelo aumento do teor do dispersante. Para os mesmos tempos, o módulo de armazenamento de energia (G') foi menor em função do aumento do teor do aditivo. Após 2 h de acompanhamento, o valor de G' das pastas aditivadas não atingiu o valor obtido na pasta pura, mostrando que a tendência natural da aglomeração do cimento foi dificultada pela ação do policarboxilato. A relação causa-efeito do processo de consolidação pode ser mais bem detalhada a partir do cruzamento dos resultados obtidos nos ensaios para acompanhamento da cinética de reação química com os obtidos quantificando a cinética de aglomeração. Assim, pode-se concluir que essa relação não é linear e, com o aumento do teor de policarboxilato, distintos níveis de aglomeração foram obtidos em semelhantes níveis de calor acumulado durante a reação de hidratação, ou seja: para se atingir os mesmos níveis de G' seriam necessários maiores tempos de reação.

\section{AGRADECIMENTOS}

Os autores agradecem à CAPES e ao CNPq pelo apoio na realização deste trabalho.

\section{REFERÊNCIAS}

[1] J. S. Lyra, Estudo da influência de policarboxilato comercial na hidratação, reologia e físico-química de superfície do cimento, Diss. Mestrado, Escola Politécnica da Universidade de S. Paulo, S. Paulo, SP (2010).

[2] V. Dodson, Concrete admixtures, Ed.:Van Nostrand Reinhold, N. York, EUA (1990) 221p.

[3] A. Griesser, Cement-Superplasticizer Interactions at Ambient Temperatures, Diss. Mestrado, Swiss Federal Institute of Technology, Zurich, Suiça (2002).

[4] J. Plank, C. H. Winter, Competitive adsorption between superplasticizer and retarder molecules on mineral binder surface, Cement Concrete Res. 38 (2008) 599-605.

[5] V. S. Ramachandran, Concrete admixtures Handbook Properties, Science, and Technology, Sec. Ed., Noyes Publ. New Jersey, EUA (1995).

[6] R. P. N. Antunes, Influência da reologia e da energia de impacto na resistência de aderência de revestimentos de argamassa, Tese Dr., Escola Politécnica da Universidade de S. Paulo, SP (2005) 162p.

[7] I. R. Oliveira, A. R. Studart, R. G. Pileggi, V. C. Pandolfelli, Dispersão e empacotamento de partículas: princípios básicos e aplicações em processamento cerâmico, Fazendo Arte Editorial, S. Paulo, SP (2000) 224 p.

[8] C. T. Hartmann, Avaliação de aditivos superplastificantes base policarboxilatos destinados a concretos de cimento Portland, Diss. Mestrado, Escola Politécnica da Universidade de S. Paulo, S. Paulo, SP (2002).

[9] F. M. Lima, Avaliação do comportamento reológico de pastas de cimento para poços de petróleo com adição de superplastificantes, Diss. Mestrado, Universidade Federal do Rio Grande do Norte, RN (2006).

[10] R. Monte, Avaliação de metodologia de ensaios destinadas à verificação da eficiência de aditivos superplastificantes em pastas de cimento Portland, Diss. Mestrado, Escola Politécnica da Universidade de S. Paulo, S. Paulo, SP (2003) 118p.

[11] F. Ridi, E. Fratini, P. Baglioni, Cement - A two thousand year old nano-colloid, J. Colloid Interf. Sci. 357, 2 (2011) 255-264.

[12] R. C. O. Romano, M. M. Takahashi, C. C. Liberato, R. G. Pileggi, Fresh and hardened characterization of airentrained cement pastes, in $13^{\text {th }}$ Int. Cong. Chem. Cement, Madrid, Espanha (2011).

[13] H. F. W. Taylor, Cement Chemistry, Academic Press Ltd., Londres, Inglaterra (1990).

[14] I. Odler, Hydration, setting and hardening of Portland cement, in P. C. Hewlett (Ed.), Lea's Chemistry of Cement 
and Concrete, 4 Ed., Arnold, Londres, Inglaterra 6 (1998) 241-297.

[15] A. Yoshimichi, F. Matsushita, S. Shibata, Y. Hama, Nano-structural changes of $C-S-H$ in hardened cement paste during drying at $50{ }^{\circ} \mathrm{C}, \mathrm{J}$. Adv. Concrete Techn. 5, 3 (2007) 313-323.

[16] J. J. Thomas, H. M. Jennings, A colloidal interpretation of chemical aging of the $C-S-H$ gel and its effects on the properties of cement paste, Cement Concrete Res. 36 (2006)
30-38.

[17] I. Jewed, J. Skalny, J. F. Young, Hydration of Portland Cement, in P. Barnes (Ed.), Structure and Performance of Cement (1983) 237-318.

[18] J. R. Garcia, I. R. Oliveira, V. C. Pandolfelli, Processos de hidratação e os mecanismos de atuação dos aditivos retardadores e aceleradores de pega do cimento de aluminato de cálcio, Cerâmica 53, 325 (2007) 42-56.

(Rec. 16/06/2011, Rev. 11/07/2011, Ac. 12/07/2011) 